Pacific Journal of Mathematics

MEASURES INVARIANT UNDER A GROUP OF 


\title{
MEASURES INVARIANT UNDER \\ A GROUP OF TRANSFORMATIONS
}

\author{
Rolando B. Chuaqui
}

The purpose of this article is to present necessary and sufficient conditions of an algebraic character for the existence of a countably additive measure defined on a $\sigma$-field of sets vanishing exactly on a given subset of the field, and invariant under a group of transformations.

0. Introduction. Most measures of interest defined on fields of sets are required to be invariant under certain groups of transformations. For any field $F$ of subsets of a set $X$, a group of transformations $G$ of $F$ is a set of one-one functions closed under composition and inverse, that contains the identity function restricted to $X$, and such that any function in $G$ transforms elements of $F$ into elements of $F$. A measure on $F$ is invariant under $G$ if any two elements $A, B \in F$, such that $A$ is the image of $B$ under a function in $G$, have the same measure.

The problem of finding algebraic conditions for the existence of such measures has been discussed in several places; in particular, in Tarski's book [14], p. 231, where necessary and sufficient conditions are given for the existence of a finitely additive measure invariant under a group of transformations. To my knowledge, no general solution of this type has been published, before this paper, for countably additive measures.

Partial solutions to this problem were obtained in [11, 2]. In this second paper a conjecture was formulated which was proved false in [3].

The proof presented here uses extensively the theory of Cardinal Algebras developed in [14]. I shall quote theorems and definitions from this book by their number followed by a T. I also use a representation theorem for certain types of Cardinal Algebras obtained in [6].

A related problem is the existence of a countably additive measure on a Boolean $\sigma$-algebra when no group of transformations is involved (for the theory of Boolean algebras see [13]). The interesting problem, in this case, is to find a strictly positive measure (i.e. a measure that vanishes only on the zero of the algebra). Necessary and sufficient conditions were found in [11], and better conditions in [10]. I shall use these latter requirements for the existence of invariant measures.

The conditions obtained for the existence of an invariant $\sigma$-measure are a combination of Kelley's requirements and the countably additive version of the main condition of Tarski for the existence of a finitely additive invariant measure: the nonexistence of paradoxical decomposi- 
tions of the unit set. The main theorem proved (Theorem 5.1) includes as particular cases results obtained by [7] for a group generated by one transformation, and by [9] for a continuous group.

Given a $\sigma$-field $\mathfrak{F}$ of sets and a group of transformations of $\mathfrak{F}$, it can be deduced from the main result in this paper mentioned above (5.1), that an invariant $\sigma$-measure exists iff an ideal in $\mathfrak{i}$ of a certain type exists. This falls short of determining algebraic necessary and sufficient conditions for the existence of an invariant $\sigma$-measure (with no predetermined ideal of null-sets). However, this shortcoming is shared by the solutions available for the existence of a $\sigma$-measure on a $\sigma$-field of sets when no group of transformations is involved: From Kelley's conditions it can also be deduced that a $\sigma$-measure on $\mathfrak{F}$ exists iff an ideal in $\mathfrak{F}$ of a certain type exists.

On the other hand, if we are given the ideal on which the measure vanishes, both Kelley's theorem and mine give algebraic necessary and sufficient conditions for its existence.

In the first section the general setting of fields of sets and groups of transformations is discussed. The second section contains some lemmas about ideals and congruence relations in Boolean Algebras and Cardinal Algebras. Section three studies the countable chain condiction. The next section gives the main theorems on invariant measures on Boolean Algebras. Finally, the fifth section applies these theorems to fields of sets.

The measure-theoretic results obtained in this paper were announced without proof in [4], where they were applied to obtain probability measures.

\section{Groups of transformations on fields of sets.} Throughout this paper we employ the usual set-theoretical terminology. We identify an ordinal number with the set of preceding ordinals, and a cardinal number with the corresponding initial ordinal. In particular $\omega$, the set of natural number is the first infinite ordinal and cardinal; $\omega_{1}$ is the first uncountable ordinal and the next cardinal after $\omega$. For functions $f, g$, we use Do $f, f^{-1}, f \circ g$, and $f * A$ respectively for the domain of $f$, the inverse of $f$, the composition of $f$ and $g$, and the image of $A$ under $f .{ }^{A} B$ denotes the set of functions from $A$ into $B$. In particular, ${ }^{\omega} A$ is the set of all denumerably infinite sequences with terms in $A$; for $n \in \omega,{ }^{n} A$ is the set of all $n$-termed sequences; ${ }^{\infty} A$ denotes the set of all finite sequences with terms in $A$. For arbitrary relations $R$, we also use $R * A$ for the image of $A$ under $R$.

We shall also study measures on Boolean algebras. By a measure on a Boolean $\sigma$-algebra (a $\sigma$-BA) $\mathfrak{B}=\langle B, V, \Lambda,-, 0,1\rangle$, we understand a countably additive, nonnegative real function on $B$ that assumes the value one at the unit of the algebra. 
If $x \in{ }^{\omega} B$, we write $V\left\{x_{1}: i \in \omega\right\}$ and $\Lambda\left\{x_{1}: i \in \omega\right\}$ for the least upper bound (l.u.b.) and greatest lower bound (g.l.b.) of the sequence $x$. A $\sigma$-field of subsets of a set $X, \mathfrak{F}=\langle F, \cup, \cap,-, \varnothing, X\rangle$, is a particular kind of $\sigma-B A$ in which the universe $F$ consists of subsets of $X$, and the operations are set-theoretic union, intersection, and complement with respect to $X$. L.u.b.'s and g.l.b.'s of denumerable sequences coincide with countable unions and intersections. A group (or more properly, a quasi-group) of transformations of the $\sigma$-field of sets $F$ is a set $G$ of one-one functions such that:

(i) If $A, B \in F, f \in G, \quad A \subseteq \operatorname{Do} f, B \subseteq \operatorname{Do} f^{-1}$, then $f * A$, $f^{-1} * B \in F$.

(ii) The identity function restricted to $X$ belongs to $G$.

(iii) If $f, g \in G$, then $f^{-1}$ and $f \circ g \in G$.

Notice that functions in $G$ are not supposed to have a common domain (see [14], p. 221).

A measure $\mu$ on $\widetilde{\mho}$ is said to be invariant under $G$, or $G$-invariant, if for any $A, B \in F$ such that there is an $f \in G$ with $A \subseteq \operatorname{Dof}$ and $B=f * A$, we have $\mu(A)=\mu(B)$. Our problem, then, is to find necessary and sufficient conditions on $\mathfrak{F}$ and $G$ for the existence of such measures. It is more convenient to work with equivalence relations on $B A$ 's thus, we define the equivalence relation $\sim_{G}$ on $F$ :

$A \sim{ }_{G} B$ iff there is an $f \in G$ such that $A \subseteq$ Do $f$ and $B=f * A$. It is clear that $\mu$ is $G$-invariant iff:

For any $A, B \in F, A \sim_{G} B$ implies $\mu(A)=\mu(B)$.

In general, for any equivalence relation $R$ on a $B A \mathfrak{B}$ and measure $\mu$ on $\mathfrak{B}$, we say that $\mu$ is $R$-invariant if for any $a, b \in B$, we have

$$
a R b \text { implies } \mu(a)=\mu(b) \text {. }
$$

If a measure $\mu$ on $F$ is $G$-invariant, then it also has to be $\simeq_{G}$-invariant for the equivalence relation on $F, \simeq_{G}$, defined by: $A \simeq{ }_{G} B$ iff there are sequences of disjoint elements $Y, Z \in{ }^{\omega} F$, such that $A=$ $\cup\left\{Y_{i}: i \in \omega\right\}, B=\cup\left\{Z_{i}: i \in \omega\right\}$, and $Y_{i} \sim{ }_{G} Z_{i}$ for every $i<\omega$.

It is easy to see, that if $\mu$ is $G$-invariant, then for any $A, B \in F$ we have,

$$
A \underset{G}{\simeq} B \text { implies } \mu(A)=\mu(B) .
$$

It is convenient to introduce the disjunctive $B A \dot{\mathfrak{B}}$ associated with a $\sigma-B A \mathfrak{B}$. Disjunctive $B A$ 's were introduced in Def. 15.14T. For any $\sigma-B A \mathfrak{B}$, the disjunctive $B A$ associated with $\mathfrak{B}$ is the partial algebra $\mathfrak{B}=\langle B,+, \Sigma\rangle$ where + is a binary partial operation and $\Sigma$ a countable partial operation defined by:

(a) For any $a, b, c \in B, a+b=c$ iff $a \vee b=c$ and $a \wedge b=0$. 
(b) For any $x \in{ }^{\omega} B$ and $c \in B, \Sigma_{i<\omega} x_{i}=c$ iff $\vee\left\{x_{i}: i \in \omega\right\}=c$ and $x_{i} \wedge x_{j}=0$ for $i<j<\omega$.

This disjunctive $B A \dot{B}$ is a generalized cardinal algebra (GCA) by 15.24T. We shall use GCA's and cardinal algebras (CA) throughout this paper. The terminology will be taken from [14] with a few exceptions that will be noted in the appropriate places.

A congruence relation $R$ on a GCA $\mathfrak{U}=\langle A,+, \Sigma\rangle$ is an equivalence relation that satisfies:

(i) if $a, b, c, d, a+b, c+d \in A ; a R c$, and $b R d$, then $a+b R c+d$;

(ii) if $x, y \in{ }^{\omega} A, \sum_{i<\omega} x_{i}, \sum_{l<\omega} y_{i} \in B$, and $x_{i} R y_{i}$ for every $i<\omega$ then $\sum_{i<\omega} x_{i} R \sum_{i<\omega} y_{i}$.

Congruence relations are called in [14], infinitely additive equivalence relations (see 6.4T).

A refining relation $R$ on a GCA $\mathfrak{U}$ is a relation on $A$ such that:

if $a, x_{0}, x_{1}, b \in A, a=x_{0}+x_{1}$, and $a R b$, then there are $y_{0}, y_{1} \in A$ such that $b=y_{0}+y_{1}, x_{0} R y_{0}$, and $x_{1} R y_{1}$.

Refining relations are called finitely refining in [14] (see 6.7T).

By $16.6 \mathrm{~T}, \simeq_{G}$ is a refining congruence relation on the GCA (disjunctive $B A$ ) $\dot{\mathfrak{F}}$.

The main purpose of this paper is to give necessary and sufficient conditions for the existence of $G$-invariant measures on $\mathfrak{\wp}$. However, it is more convenient to work in a more general setting and find measures $\mu$ on a $\sigma-B A, \mathfrak{B}$ that are $R$-invariant for $R$ a refining congruence relation on $\mathfrak{B}$. I shall deal with this problem in the following sections, returning to $\mathfrak{F}$ in the last section.

2. Ideals and congruence relations. In this section I shall prove some lemmas, which will be needed later, about ideals and congruence relations in $\sigma-B A$ 's and GCA's. For any $\sigma-B A \mathfrak{B}$ we have the corresponding disjunctive $B A \dot{\mathfrak{B}}$, which is a GCA. The notion of an ideal in a GCA is defined and discussed in ([14], Chapter 9). I shall call ideals in a GCA cardinal ideals to distinguish them from ideals in a $B A$ (see [13] for ideals in $B A$ 's). There are, then, two notions of ideals in $\mathfrak{B}$ : $\sigma$-ideals in $\mathfrak{B}$ as a $B A$ and cardinal ideals in $\dot{B}$ as a GCA. The first lemma proves that they coincide.

Lemma 2.1. Let $\mathfrak{B}$ be a $\sigma-B A, I \subseteq B$. Then, $I$ is a $\sigma$-ideal in $\mathfrak{B}$ iff $I$ is a cardinal ideal in $\dot{\mathfrak{B}}$.

Proof. Suppose $I$ is a cardinal ideal in $\dot{\mathfrak{B}}$. It is clear that the partial 
ordering in $\mathfrak{B}$ coincides with that in $\dot{\mathfrak{B}}$. Thus, if $a \in I$ and $b \leqq a$ (in $\mathfrak{B}$ ), then $b \in I$. Suppose, now that $x \in{ }^{\omega} I$. Define

$$
\begin{aligned}
& x_{0}^{\prime}=x_{0} \\
& x_{n+1}^{\prime}=x_{n+1}-\vee\left\{x_{i}: i \leqq n\right\}
\end{aligned}
$$

It is clear that $x^{\prime} \in{ }^{\omega} I$ and $x_{i}^{\prime} \wedge x_{j}^{\prime}=0$ for $i \neq j$. Then,

$$
\vee\left\{x_{i}: i \in \omega\right\}=\sum_{i<\omega} x_{i}^{\prime} \in I \text {. }
$$

Thus, $I$ is a $\sigma$-ideal in $\mathfrak{B}$.

The converse implication is obvious.

The next lemma proves that the equivalence relation determined by a $\sigma$-ideal $I$ on a $\sigma$-BA $\mathfrak{B}$ is the same as the relation determined by $I$ as a cardinal ideal in $\dot{\mathfrak{B}}$ (cf. 9.26T).

Lemma 2.2. Let $\mathfrak{B}$ be a $\sigma-B A, I$ a $\sigma$-ideal in $\mathfrak{B}$. Then:

(i) for any $a, b \in B, a-b \vee b-a \in I$ iff there are $a^{\prime}, b^{\prime} \in I$ and $c \in B$ such that $a=c+a^{\prime}$ and $b=c+b^{\prime}$;

(ii) $(\dot{B} / I)=\dot{\mathfrak{B}} / I$, i.e. if $x \in{ }^{\omega} B, c \in B$, then $\Sigma_{l<\omega}\left(x_{i} / I\right)=c / I$ iff there is an $x^{\prime} \in{ }^{\omega} B$ such that $x_{i}^{\prime} \wedge x^{\prime}=n \quad \vee / I=r^{\prime} / I$ and $S \quad(r / I)=$ $\left(\sum_{1<\omega} x_{i}^{\prime}\right) / I$, for $i<j<\omega$.

Proof. (i) (1) Suppose $a-b \vee b-a \in I$. Take $a^{\prime}=a-b, b^{\prime}=$ $b-a, c=a \wedge b$.

(2) Suppose $a=a^{\prime}+c, \quad b=b^{\prime}+c, \quad$ and $\quad b^{\prime}, a^{\prime} \in I$. Then $a-b \vee b-a \leqq a^{\prime} \vee b^{\prime} \in I$.

(ii) Suppose $\sum_{i<\omega}\left(x_{i} / I\right)=c / I$. Then $x_{i} / I \wedge x_{j} / I=0 / I$ and so, $x_{i} \wedge x_{j} \in I$ for $i<j<\omega$. Let $d=\vee\left\{x_{i} \wedge x_{j}: i<j<\omega\right\}$ and $x_{i}^{\prime}=x_{i}-d$ for $i<\omega$. Then $d \in I, \quad x_{\imath}^{\prime} \wedge x_{j}^{\prime}=0, \quad x_{i}^{\prime} / I=x_{i} / I, \quad$ and $\quad \sum_{i<\omega}\left(x_{i}^{\prime} / I\right)=$ $\left(\sum_{i<\omega} x_{i}^{\prime}\right) / I=c / I$ for $i<j<\omega$.

We are interested in refining congruence relations on disjunctive $B A$ 's $\dot{\mathfrak{B}}$, and how they behave when we pass to $\dot{\mathfrak{B}} / I$ for an ideal $I$. This is given in the following lemma:

LEMMA 2.3. Let $\mathfrak{B}$ be a $\sigma-B A, I$ a $\sigma$-ideal on $\mathfrak{B}$, and $R$ a refining congruence relation on $\dot{B}$ such that $R * I \subseteq I$ (i.e. if $a \in I$ and $a R b$, then $b \in I$ ). Define $\bar{R}$ on $\dot{\mathfrak{B}} / I$ by:

$a / I \bar{R} b / I$ iff there are $a^{\prime}, b^{\prime} \in B$ such that $a / I=a^{\prime} / I$, $b / I=b^{\prime} / I$, and $a^{\prime} R b^{\prime}$. 
Under these conditions, $\bar{R}$ is a refining congruence relation on $\dot{\mathfrak{B}} / \mathbf{I}$.

Proof. It is easy to see that $\bar{R}$ is an equivalence relation on $B$. We have to prove that $\bar{R}$ is refining and preserves $\Sigma$. Let

(1) $a / I \bar{R} b / I$

(2) $a / I=x_{1} / I+x_{2} / I$.

By (2) and 2.2(ii), there are disjoint $x_{1}^{\prime}, x_{2}^{\prime}$ such that

(3) $x_{1} / I=x_{1}^{\prime} / I$ and $x_{2} / I=x_{2}^{\prime} / I$.

Also, by (1), there are $a^{\prime}, b^{\prime}$ such that $a / I=a^{\prime} / I, b / I=b^{\prime} / I$, and $a^{\prime} R b^{\prime}$. By (3), $a^{\prime} / I=\left(x_{1}^{\prime}+x_{2}^{\prime}\right) / I$. Thus, there are $x_{1}^{\prime \prime}, x_{2}^{\prime \prime}$ such that,

(4) $a^{\prime}=x_{1}^{\prime \prime}+x_{2}^{\prime \prime}, x_{1} / I=x_{1}^{\prime \prime} / I$, and $x_{2} / I=x_{2}^{\prime \prime} / I$.

Since $R$ is refining and (4), $b^{\prime}=y_{1}+y_{2}, x_{1}^{\prime \prime} R y_{1}$, and $x_{2}^{\prime \prime} R y_{2}$. Then, $b / I=y_{1} / I+y_{2} / I, y_{1} / I \bar{R} x_{1} / I$, and $y_{2} / I \bar{R} x_{2} / I$. Thus, $\bar{R}$ is refining.

Suppose now that $x, y \in{ }^{\omega} B$ with,

(5) $x_{i} / I \bar{R} y_{i} / I$ for all $i<\omega$;

(6) $\sum_{i<\omega}\left(x_{i} / I\right)$ and $\sum_{i<\omega}\left(y_{i} / I\right)$ exist in $B / I$. From (5) we obtain $x^{\prime}, y^{\prime} \in{ }^{\omega} B$ such that,

(7) $x_{i}^{\prime} / I=x_{i d} / I, y_{i}^{\prime} / I=y_{i} / I$, and $x_{i}^{\prime} R y_{i}^{\prime}$, for all $i<\omega$.

From (6) we deduce that $x_{i} / I \wedge x_{j} / I=0 / I=y_{i} / I \wedge y_{j} / I$ for all $i, j$, with $i<j<\omega$. Then, the same is true for $x^{\prime}, y^{\prime}$. Thus

(8) $x_{i}^{\prime} \wedge x_{j}^{\prime} \in I$, for all $i, j<\omega, i \neq j$.

Take $c=\vee\left\{x_{i}^{\prime} \wedge x_{j}^{\prime}: i<j<\omega\right\}$. Then $c \in I$. Define $x_{i}^{\prime \prime}=$ $x_{i}^{\prime}-c$. Then $x_{i}^{\prime \prime} / I=x_{\imath}^{\prime} / I=x_{i} / I$. Also,

(9) $x_{i}^{\prime \prime} \wedge x_{j}^{\prime \prime \prime}=0$ for $i<j<\omega$.

We have, $x_{i}^{\prime}=x_{i}^{\prime \prime}+z_{i}$ where $z_{i}=c \wedge x_{i}^{\prime}$ and $z_{i} \in I$. From (7) we obtain $y^{\prime \prime}, z^{\prime} \in{ }^{\omega} B$ such that,

(10) $y_{i}^{\prime}=y_{i}^{\prime \prime}+z_{i}^{\prime}, y_{i}^{\prime \prime} R x_{i}^{\prime \prime}$, and $z_{i}^{\prime} R z_{i}$.

Since $R * I \subseteq I, z_{i}^{\prime} \in I$ and

(11) $y_{i}^{\prime \prime} / I=y_{i}^{\prime} / I=y_{i} / I$.

Similarly as (7) we get,

(12) $y_{i}^{\prime \prime} \wedge y_{j}^{\prime \prime} \in I$ for $i<j<\omega$.

Take $d=\vee\left\{y_{i}^{\prime \prime} \wedge y_{j}^{\prime \prime}: i<j<\omega\right\}(\in I)$ and $y_{i}^{\prime \prime \prime}=y_{i}^{\prime \prime}-d$. We have,

(13) $y_{i}^{\prime \prime \prime} / I=y_{i}^{\prime \prime} / I=y_{i} / I$ and $y_{i}^{\prime \prime \prime} \wedge y_{j}^{\prime \prime \prime}=0$ for $i<j<\omega$. Also, $y_{i}^{\prime \prime}=$ $y_{i}^{\prime \prime \prime}+u_{i}$ where $u_{i}=d \wedge y_{i}^{\prime \prime} \in I$. From (9) we obtain,

(14) $x_{i}^{\prime \prime}=x_{i}^{\prime \prime \prime}+u_{i}^{\prime}, x_{i}^{\prime \prime \prime} R y_{i}^{\prime \prime \prime}$, and $u_{i}^{\prime} R u_{i}$ for all $i<\omega$. Thus, since $R * I \subseteq I, u_{i}^{\prime} \in I$ and,

(15) $x_{i}^{\prime \prime \prime} / I=x_{i}^{\prime \prime} / I=x_{i} / I$ for all $i<\omega$.

Since $x_{i}^{\prime \prime \prime} \leqq x_{i}^{\prime \prime},(9)$ implies:

(16) $\quad x_{i}^{\prime \prime \prime} \wedge x_{j}^{\prime \prime \prime}=0$ for $i<j<\omega$.

Since $R$ is a congruence relation on $\dot{\mathfrak{B}},(13),(14)$, and (16) imply that $\left(\sum_{i<\omega} x_{i}^{\prime \prime \prime}\right) R\left(\sum_{1<\omega} y_{i}^{\prime \prime}\right)$. But then from (15) and (13) we obtain the desired conclusion; i.e. 
The following lemma relates cardinal ideals in the GCA's $\mathfrak{A}$ and $\mathfrak{U} / R$.

LEMMA 2.4. Let $\mathfrak{A}$ be a GCA, $R$ a refining congruence relation on $\mathfrak{A}, I \subseteq A$, and $R * I \subseteq I$. Then $I$ is a cardinal ideal in $\mathfrak{A}$ iff $I / R$ is a cardinal ideal in $\mathfrak{U} / R$.

Proof. (1)Suppose $I$ is a cardinal ideal in $\mathfrak{A}$. Let $a / R \in I / R$, $a \in I$, and $b / R \leqq a / R$. Then, there are $a^{\prime}, b^{\prime} \in A$ with $b^{\prime} \leqq a^{\prime}, a R a^{\prime}$, and $b R b^{\prime}$. Thus, since $R * I \subseteq I$, we have that $a^{\prime}, b^{\prime} \in I$. But, then, $b^{\prime} / R=b / R \in I / R$.

Let, now, $x \in{ }^{\omega} I$ and $\Sigma_{1<\omega}\left(x_{i} / R\right) \in A / R$, i.e. $\Sigma_{i<\omega}\left(x_{i} / R\right)=b / R$ for some $b \in A$. Then, there are $x^{\prime} \in{ }^{\omega} A$, and $b^{\prime} \in A$ such that $\sum_{i<\omega} x_{i}^{\prime}=b^{\prime}$, $x_{i} R x_{\imath}^{\prime}$, and $b R b^{\prime}$, for all $i<\omega$. Since $R * I \subseteq I, x_{\imath}^{\prime} \in I$ for $i<\omega$. Then $\sum_{i<\omega} x_{i}^{\prime} \in I$ and, thus $b / R=b^{\prime} / R \in I / R$.

(2) Suppose, now, that $I / R$ is a cardinal ideal in $\mathfrak{A} / R$. Let $a \in I$, and $b \leqq a$. Then $a / R \in I / R$, and $b / R \leqq a / R$. Thus, $b / R \in I / R$, i.e. there is a $b^{\prime} \in I$ such that $b^{\prime} R b$. But, then from $R * I \subseteq I$, we get $b \in I$.

Suppose $x \in{ }^{\omega} I$ with $\Sigma_{i<\omega} x_{i} \in A$. Then $x_{i} / R \in I / R$ for all $i<\omega$, and $\sum_{i<\omega}\left(x_{i} / R\right) \in I / R$. Thus, $\sum_{i<\omega}\left(x_{i} / R\right)=\left(\sum_{i<\omega} x_{i}\right) / R \in I / R$. Therefore, there is a $b \in I$ such that $\sum_{i<\omega} x_{\imath} R b$. But then, from $R * I \subseteq I$, we get that $\sum_{i<\omega} x_{i} \in I$.

3. The countable chain condition. Let $\mathfrak{A}$ be a GCA. We say that a subset $B \subseteq A$ is bounded if there is an $a \in A$ such that $b \leqq a$ for every $b \in B$. $\mathfrak{U}$ satisfies the countable chain condition $(c c c)$ if every bounded subset $B \subseteq A$ well ordered by the relation $\leqq$ is at most countable. It is clear that if $\mathfrak{B}$ is a $\sigma-B A$, then the GCA $\dot{B}$ satisfies the $c c c$ if and only if $B$ satisfies the countable chain condition in the usual Boolean sense. [5] calls a GCA that satisfies the $c c c$, separable. In this section I shall prove that the $c c c$ is transmitted through several constructions of GCA's.

If $a, b \in A$, we write $a \wedge b, a \vee b$ for the g.l.b. and l.u.b. of $a, b$; if $x \in{ }^{\omega} A, \wedge_{i \in \omega} x_{i}$ and $\vee_{i \in \omega} x_{i}$ stand for the g.l.b. and l.u.b. of the sequence $x$ (see Defs. 3.1T and 3.2T where slightly different symbols are employed).

THEOREM 3.1. Let $\mathfrak{A}$ be a GCA satisfying the ccc, and $R$ a refining, congruence relation on $\mathfrak{A}$. Then $\mathfrak{U} / R$ also satisfies the ccc.

Proof. Suppose the sequence $y \in{ }^{\omega_{1}}(A / R)$ is such that for $\beta<\alpha<$ $\omega_{1}$, we have $y_{\beta}<y_{\alpha} \leqq a / R$ for some $a \in A$. Choose a sequence $x \in$ ${ }^{\omega_{1}} A$, such that $y_{\alpha}=x_{\alpha} / R$ and $x_{\alpha} \leqq a$ for every $\alpha \in \omega_{1}$. It is possible to obtain such an $x$ because $R$ is refining.

We shall first prove: 
(1) For any $b, c \in A$ with $b, c \leqq a$, we have $b / R<c / R$ iff there is a $d \in A$ such that $d \leqq a, b<d$, and $d R c$.

From $b / R<c / R$ we get $b^{\prime}$ with $b^{\prime}<c$ and $b R b^{\prime}$. Thus, we have, $b^{\prime}<c \leqq a, b \leqq a, b R b^{\prime}$, and $a R a$. Using 7.13T (vi) we obtain a $d$ with $b \leqq d \leqq a$ and $d R c$. Now, if $b=d$, we would have $c / R=d / R=b / R$ contradicting $b / R<c / R$. Thus $b<d$.

The converse implication is proved similarly, and, thus, (1) is proved. We pass now, to the proof of the theorem:

Define by transfinite recursion the sequence $z \in{ }^{\omega_{1}} A$ satisfying:

(2) $y_{\alpha}=x_{\alpha} / R=z_{\alpha} / R$, and if $\alpha<\beta<\omega$, then $z_{\alpha}<z_{\beta} \leqq a$, as follows:

(a) $z_{0}=x_{0}$.

(b) Suppose $z_{\alpha}$ is defined. We have $z_{\alpha} / R<x_{\alpha+1} / R$, and $z_{\alpha}, x_{\alpha+1} \leqq$ a. By (1) there is $d \in A$ such that $z_{\alpha}<d \leqq a$, and $d R x_{\alpha+1}$. Take $z_{\alpha+1}=d$.

(c) Let $\alpha$ be a limit ordinal, $\alpha<\omega_{1}$, and $z_{\beta}$ defined for all $\beta \in \alpha$. Let $f \in{ }^{\omega} \alpha$ be a strictly increasing sequence of ordinals cofinal in $\alpha$. We shall prove that

(3) $\left(\vee_{i \in \omega} z_{f(i)}\right) / R=\vee_{\beta \in \alpha} y_{\beta}$.

It is enough to prove,

$$
\left(\bigvee_{i \in \omega} z_{f(i)}\right) / R=\bigvee_{i \in \omega}\left(z_{f(i)} / R\right)
$$

Let $z_{f(i)}+u_{i}=z_{f(i+1)}$ for every $i<\omega$. Then

$$
z_{f(i)} / R+u_{i} / R=z_{f(i+1)} / R \quad \text { for every } i<\omega .
$$

Also, $\vee_{i<\omega} z_{f(l)}=z_{f(0)}+\sum_{i<\omega} u_{i}$. Thus,

$$
\begin{aligned}
\left(\vee_{i \in \omega} z_{f(i)}\right) / R & =z_{f(0)} / R+\sum_{i<\omega}\left(u_{i} / R\right) \\
& =\vee_{i<\omega}\left(z_{f(i)} / R\right) .
\end{aligned}
$$

So (3) is proved.

Let now, $b=\vee_{i \in \omega} z_{f(i)}$. Then, since $b / R=\vee_{\beta \in \alpha} y_{\beta} \leqq y_{\alpha}$, and $b \leqq a$, by (1) there is a $d \in A$ with $b \leqq d \leqq a$ and $d R x_{\alpha}$. Let, then, $z_{\alpha}=d$.

With this, we complete definition (2) and, thus, obtain a $\leqq-$ wellordered bounded subset of $A$ of type $\omega_{1}$, contradicting the $c c c$ for $\mathfrak{A}$.

We pass now, to show preservation of the $c c c$ under another important operation on GCA's: the cardinal product (see Def. 6.11T).

THEOREM 3.2. Let $\mathfrak{A}$, be GCA's that satisfy the ccc for every $i \in I$. Then $\Pi_{i \in I} \mathfrak{A}_{i}$ also satisfies the ccc. 
Proof. Suppose $y \in{ }^{\omega_{i}}\left(\prod_{i \in I} A_{i}\right)$ and $a \in \Pi_{i \in I} A_{i}$ be such that $y_{\beta} \leqq$ $y_{\alpha} \leqq a$ for $\beta<\alpha<\omega_{1}$. We then have $y_{\beta i} \leqq y_{\alpha i} \leqq a_{i}$ for every $i \in I$.

The sets $B_{i}=\left\{y_{\alpha}: \alpha \in \omega_{1}\right\} \subseteq A_{\imath}$ are bounded and $\leqq$-well-ordered for all $i \in I$; then, card $\left(B_{\imath}\right) \leqq \omega$ for all $i \in I$.

Since $\operatorname{card}\left(B_{i}\right) \leqq \omega$ for every $i \in I$, there is an $\alpha \in \omega_{1}$ such that $y_{\alpha t}=y_{\beta i}$ for all $\beta \geqq \alpha$. Define $\gamma(i)$ as the least such $\alpha$, i.e.,

$$
\gamma(i)=\cap\left\{\alpha: y_{\alpha i}=y_{\beta t} \text { for all } \beta \geqq \alpha\right\} .
$$

We have, $\gamma(i) \in \omega_{1}$.

Let $J=\left\{i: a_{i} \neq 0_{l}\right\}$. From the definition of cardinal product, $\operatorname{card}(J) \leqq \omega$. Also, for every $\alpha \in \omega_{1}, y_{\alpha i}=0_{i}$ for all $i \in I-J$. Let $\delta$ be the least upper bound of $\gamma(i)$ for $i \in J$; i.e.

$$
\delta=\cup\{\gamma(i): i \in J\} .
$$

$\delta \in \omega_{1}$, and $y_{\alpha}=y_{\delta}$ for all $\alpha \geqq \delta$. Therefore, $\operatorname{card}\left\{y_{\alpha}: \alpha \in \omega_{1}\right\}=\omega$.

COROLlary 3.3. Let $\mathfrak{A}$ be a GCA that satisfies the ccc. Then " $\mathfrak{A}$ also satisfies the ccc.

Every GCA $\mathfrak{A}$ can be closed to obtain a CA $\overline{\mathfrak{A}}$ which preserves most of the properties of $\mathfrak{A}$. In [14, Ch. 7], these closures are studied. We prove, now, that closures preserve the $c c c$.

THEOREM 3.4. Let $\mathfrak{A}$ be a GCA that satisfies the ccc. Then $\overline{\mathfrak{A}}, a$ closure of $\mathfrak{X}$, also satisfies the ccc.

Proof. By 7.7T, $\overline{\mathfrak{A}}$ is isomorphic to ${ }^{\omega} \mathfrak{Q} / R$ where $R$ is a refining congruence relation in $\mathfrak{A}$. Thus, from 3.1 and 3.3 we obtain 3.4.

COROLlaRY 3.4. Let $\mathfrak{B}$ be a $\sigma$-BA that satisfies the ccc, $R$ a refining congruence relation on $\mathfrak{B}$, and $\mathfrak{U}=\overrightarrow{\mathfrak{B} / R}$ (a closure of $\dot{\mathfrak{B}} / R)$. Then $\mathfrak{A}$ is a CA such that for any $x \in{ }^{\omega} A$ we have $\wedge_{t \in \omega} x_{i} \in A$.

Proof. $\quad \mathfrak{A}$ satisfies the $c c c$ by 3.1 and 3.4. Hence applying 3.35T we obtain the conclusion.

4. Invariant measures in Boolean algebras. In this section we prove some theorems about the existence of $R$-invariant measures on a $\sigma$-distributive $\sigma$-BA $\mathfrak{B}$ where $R$ is a refining congruence relation on $\dot{B}$. In the next section we apply the theorems to obtain $G$-invariant measures on $\sigma$-fields of sets.

For some of the following definitions see [10, 13 p.p. 62, 204]. 
Let $\mathfrak{B}$ be a $\sigma-B A$ and $x \in{ }^{n} B$ for some $n \in \omega$. We define $i(x)=$ $m / n$ where $m$ is the largest integer $k \leqq n$ such that

$$
x_{i \mathrm{~b}} \wedge \cdots \wedge x_{i_{k-1}} \neq 0 \text { for } 0 \leqq i_{0}<i_{1}<\cdots<i_{k-1}<n \text {. }
$$

Then, if $A \subseteq B$ we define the intersection number of $A$ :

$$
i(A)=\inf \left\{i(x): x \in{ }^{n} A \text { for some } n \in \omega\right\} .
$$

We say that $\mathfrak{B}$ has the Kelley property if $B-\{0\}$ is a countable union of sets with positive intersection number. $\mathfrak{B}$ is $\sigma$-distributive if for every double sequence $x \in{ }^{\omega \times \omega} B$ we have,

$$
\vee\left\{\wedge\left\{x_{i j}: j \in \omega\right\}: i \in \omega\right\}=\wedge\left\{\vee\left\{x_{i, \phi(i)}: i \in \omega\right\}: \phi \in{ }^{\omega} \omega\right\}
$$

We say that $\mathfrak{B}$ is weakly $\sigma$-distributive if (*) is satisfied for every double sequence $x \in{ }^{\omega \times \omega} B$ such that $x_{i, j+1} \leqq x_{i j}$ for every $i, j \in \omega$. have,

Let $I \subseteq B$ and $\mu$ a measure on $\mathfrak{B}$. We say that $\mu$ is $I$-positive if we

$$
\mu(a)=0 \quad \text { iff } \quad a \in I, \quad \text { for every } a \in B .
$$

Let $a \in B$; we say that $a$ is $R$-negligible if there is a sequence of disjoint elements of $B, x \in{ }^{\omega} B$, such that $x_{i} R a$ for every $i \in \omega$. Let $N_{R}=\{a: a$ is $R$-negligible $\}$. It is clear that if the measure $\mu$ is $R$-invariant, then if $a \in N_{R}$, we must have $\mu(a)=0$.

We need the following lemma about $N_{R}$ :

LEMMA 4.1. Let $\mathfrak{B}$ be a $\sigma-B A$, and $R$ a refining congruence relation on $\dot{\mathfrak{B}}$. Then $N_{R}$ is a $\sigma$-ideal in $\mathfrak{B}$.

Proof. By 2.1 it is enough to prove that $N_{R}$ is a cardinal ideal in $\dot{\mathfrak{B}}$. Consider $\mathfrak{A}=\dot{\mathfrak{B}} / R ; \mathfrak{A}$ is a GCA. Let $h=1 / R$ and

$$
A(h)=\{c \in \mathfrak{A}: c+h=h\} .
$$

Then, by $9.15 \mathrm{~T}, A(h)$ is a cardinal ideal. It is easy to see that $R *\left(N_{R}\right) \subseteq N_{R}$. Hence by 2.4 it is enough to prove that $A(h)=N_{R} / R$.

Since $c \in A(h)$ iff $\infty c \leqq h$ (by 1.29T) the proof is reduced to:

$$
a \in N_{R} \quad \text { iff } \quad \infty(a / R) \leqq h .
$$

Suppose, first, that $a \in N_{R}$. Let $x \in{ }^{\omega} B$ be such that $x_{i} \wedge x_{j}=0$ and $x_{i} R a$ for every $i<j<\omega$. 
We have, $a / R=x_{i} / R$, for every $i<\omega$. But $\sum_{i<\omega} x_{i} \leqq 1$. Thus, $\infty(a / R)=\sum_{i<\omega}\left(x_{i} / R\right) \leqq 1 / R=h$.

Suppose, now, that $\infty(a / R) \leqq h$. Since $h \in \mathfrak{A}, \infty(a / R) \in \mathfrak{U}$ by 7.4T. Then, $\infty(a / R)=b / R$ for some $b \in B$. From the definition of the coset algebra (Def. 6.3T), there is a sequence of disjoint elements $x \in{ }^{\omega} B$ and a $c \in B$ such that $\Sigma_{i<\omega} x_{i}=c$ and $x_{i} R a$ for all $i<\omega$. Thus, $a \in N_{R}$.

We formulate, now, the main theorem of this section:

THEOREM 4.2. Let $\mathfrak{B}$ be a $\sigma$-distributive $\sigma-B A, R$ a refining congruence relation on $\dot{B}$, and $I$ a subset of $B$. The following conditions are necessary and jointly sufficient for the existence of a countably additive, $I$-positive, and $R$-invariant measure on $\mathfrak{B}$ :

(i) $\quad I$ is a proper $\sigma$-ideal;

(ii) $N_{R} \subseteq I$;

(iii) $R * I \subseteq I$;

(iv) $\mathfrak{B} / I$ has the Kelley property and is weakly $\sigma$-distributive.

For the proof we need a result of [6]. We have to introduce some definitions. Let $X$ be the Stone space of a $\sigma-B A$ and $\overline{\mathbf{R}}$ the nonnegative real numbers with $\infty$. Then $\mathscr{C}(X, \overline{\mathbf{R}})$ denotes the set of continuous functions on $X$ with compact support and values in $\overline{\mathbf{R}}$. $\langle\mathscr{C}(X, \overline{\mathbf{R}}),+, \Sigma\rangle$ is a $C A$ (cf [6], p. 31) where + is pointwise addition, and $\sum_{i<\omega} f_{\imath}$ is the continuous limit of the partial sums $\sum_{i<n} f_{i}$. This limit differs from the pointwise limit in a set of first category.

Proof of 4.2. The necessity of the conditions is easy to prove. We must use Kelley's necessary and sufficient condition for the existence of a strictly positive measure on a $B A$ (see [10], Th. 9, and [8], Th. 3.7).

We proceed, now, to construct the desired measure. For the rest of this section let $\mathfrak{B}, R$, and $I$ satisfy (i)-(iv). Define the relation $\bar{R}$ on $\mathfrak{B} / I$ by:

$a / I \bar{R} b / I$ iff these are $a^{\prime}, b^{\prime} \in B$ such that $a / I=a^{\prime} / I$,

$$
b / I=b^{\prime} / I, \quad \text { and } \quad a^{\prime} R b^{\prime}
$$

By $2.3, \bar{R}$ is refining congruence relation on $\dot{\mathfrak{B}} / I$. Let $\mathfrak{A}=(\overline{\mathfrak{B} / I) / \bar{R}}$, i.e. is a CA that is a closure of the GCA $(\dot{B} / I) / \bar{R}$.

Let $h=(1 / I) / \bar{R}$. We shall prove that:

LEMMA 4.3. $h$ is finite (Def. 4.10T: $h+a=h$ implies that $a=0$ for all $a \in A)$. In fact, we have that $h+a=h$ and $a=c / I / \bar{R}$, implies that $c / I=0 / I$. 
Proof. Suppose $h+a=h$. Then by $1.29 \mathrm{~T}, \infty a \leqq h$. Let $a=$ $(c / I) / \bar{R}$ with $c \in B$. By the refining property of $\bar{R}$ and 2.2 we obtain a sequence of disjoint elements $x \in{ }^{\omega} B$ such that $c / I \bar{R} x_{1} / I$ for all $i<$ $\omega$. From the definition of $\bar{R}$ we get $x^{\prime}, z \in{ }^{\omega} B$ such that $c / I=z_{l} / I$, $x_{i} / I=x_{i}^{\prime} / I$, and $z_{i} R x_{i}^{\prime}$ for every $i<\omega$. Thus $c=u_{i}+v_{t}, z_{i}=u_{i}+t_{i}$, $x_{i}=y_{i}+y_{i}^{\prime}$, and $x_{i}^{\prime}=y_{i}+y_{i}^{\prime \prime} \quad$ with $v_{i}, t_{i}, y_{i}^{\prime}, y_{i}^{\prime \prime} \in I$. Let $d=$ $\wedge\left\{u_{i}: i \in \omega\right\}$. Then as $\mathfrak{B}$ is $\sigma$-distributive, $c=d+e$ with $e \in I$. Now, $d R s_{i}$ with $s_{i} \leqq x_{\imath}^{\prime}$ for every $i<\omega$. Thus, by $2.4 \mathrm{~T}, s_{i}=s_{i}^{\prime}+s_{\imath}^{\prime \prime}$ with $s_{1}^{\prime} \leqq y_{i}$ and $s_{i}^{\prime \prime} \leqq y_{i}^{\prime \prime}$. Then, $s_{i}^{\prime \prime} \in I$ for every $i<\omega$. Therefore, $d=r_{i}+r_{i}^{\prime}$ with $r_{i} R s_{i}^{\prime}$ and $r_{i}^{\prime} R s_{i}^{\prime \prime}$. Since $R * I \subseteq I, r_{i}^{\prime} \in I$ for every $i<\omega$. Thus by $\sigma$-distributivity, $d=\wedge\left\{r_{i}: i \in \omega\right\}+f$ where $f \in I$. We have that $s^{\prime}$ is a sequence of disjoint elements of $B$. Thus, $\wedge\left\{r_{i}: i \in \omega\right\} R p_{i} \leqq s_{i}^{\prime}$ for every $i<\omega$; so, $\wedge\left\{r_{i}: i \in \omega\right\}$ is $R$-negligible and, therefore it belongs to I. Then $d \in I$ and $c \in I$. Therefore, $c / I=0 / I$ and $a=0$. Thus, we have proved 4.3 .

$\mathfrak{U}$, also, has the following two properties:

(1) $a \wedge b \in A$, for every $a, b \in A$.

This is obtained from 3.4 , because $\mathfrak{B} / I$ satisfies the $c c c$.

(2) For every $a \in A, a \leqq \infty h$.

(2) is obtained from 7.1T.

Let $\mathbb{E}$ be the $\sigma-B A$ of idemmultiple elements of $\mathfrak{A}$ ( $a$ is idemmultiple if $a=a+a$; see Def. 4.1T and 8.3T for this algebra). Let $X$ be the Stone space of $\mathbb{F}$. 3.11 of Fillmore 1965 implies that $\mathfrak{A}$ is isomorphic to a subalgebra of $\langle\mathscr{C}(X, \overline{\mathbf{R}}),+, \Sigma\rangle$, say by a function $F$. For each element $a \in A, F(a)$ has support $E(a)$, the open-closed set corresponding to $\infty a$. Also, $F(h)$ is the characteristic function of $E(h)=X$.

There is a strictly positive measure $\mu$ on $\&$ with $\mu(\infty h)=1$. In order to prove this, we need the following two lemmas:

LEMMA 4.4. If $\mathfrak{B} / I$ has the Kelley property, then $\&$ also has the Kelley property.

Proof. Suppose $B / I-\{0 / I\}=\cup\left\{B_{n}: n \in \omega\right\}$ where each $B_{n}$ has a positive intersection number. Let,

$$
E_{n}=\left\{a: a \in E \text { and there is a } b \in B_{n} \text { such that } b / \bar{R} \leqq a\right\} .
$$

In this definition, $\leqq$ is the partial ordering of $\mathfrak{A}$.

Let $x \in{ }^{n} E_{m}$ and $y \in{ }^{n} B_{m}$ be such that $y_{k} / \bar{R} \leqq x_{k}$ for all $k<n$. Let $i(x)=m_{x} / n$ where $m_{x}$ is the largest $k$ such that

$$
x_{i_{0}} \wedge \cdots \wedge x_{i_{k-1}} \neq 0 \quad \text { for } \quad i_{0}<\cdots<i_{k-1}<n ; \quad i(y)=m_{y} / n
$$

where $m_{y}$ is defined similarly. We shall prove that $m_{x} \geqq m_{y}$. 
Suppose that $k \leqq m_{y}$. Then, there are $y_{i o}, \cdots, y_{i k-1}$ such that $y_{i_{0}} \wedge \cdots \wedge y_{i_{k-1}} \neq 0$. Thus,

$$
\infty\left(y_{w_{0}} \wedge \cdots \wedge y_{i_{k-1}}\right) / \bar{R} \neq 0 .
$$

But

$$
\begin{aligned}
\infty\left(y_{i_{0}} \wedge \cdots \wedge y_{i_{k-1}}\right) / \bar{R} & \leqq \infty\left(y_{i_{0}} / \bar{R}\right) \wedge \cdots \wedge \infty\left(y_{i_{k-1}} / \bar{R}\right) \\
& \leqq x_{i_{0}} \wedge \cdots \wedge x_{i_{k-1}}
\end{aligned}
$$

so, $x_{i 0} \wedge \cdots \wedge x_{i k-1} \neq 0$.

Thus, we have proved that $i(x) \geqq i(y)$. But

$$
i\left(E_{m}\right)=\inf \left\{i(x): x \in{ }^{\omega} E_{m}\right\} \geqq \inf \left\{i(y): y \in{ }^{\omega} B_{m}\right\}=i\left(B_{m}\right)>0 \text {. }
$$

Therefore, the lemma is proved.

LEMMA 4.5. If $\mathfrak{B} / I$ is weakly $\sigma$-distributive, then $\mathcal{E}$ is also weakly $\sigma$-distributive.

Proof. In ([13], Th. 30.1 (2)), it is proved that a $\sigma-B A$ is weakly $\sigma$-distributive iff for every $x \in{ }^{\omega \times \omega} E$ we have that:

$$
\wedge\left\{\vee\left\{x_{t s}: s \in \omega\right\}: t \in \omega\right\} \neq 0
$$

implies that there is a function $\phi$ from $\omega$ into the finite subsets of $\omega$ such that,

$$
\wedge\left\{\vee\left\{x_{t s}: s \in \phi(t)\right\}: t \in \omega\right\} \neq 0
$$

Suppose, then, that $x \in{ }^{\omega \times \omega \mathscr{E}}$ and

$$
\wedge\left\{\vee\left\{x_{t s}: s \in \omega\right\}: t \in \omega\right\} \neq 0
$$

So, there is a $c \in B / I, c \neq 0$, such that,

$$
c / \bar{R} \leqq \wedge\left\{\vee\left\{x_{t s}: s \in \omega\right\}: t \in \omega\right\} .
$$

Since the elements $x_{t s}$ of $\mathscr{E}$ are idemmultiple in $\mathfrak{A}$, by $4.7 \mathrm{~T}$ we get,

$$
c / \bar{R} \leqq \sum_{s \in \omega} x_{t s}, \quad \text { for every } t \in \omega .
$$

From $2.2 \mathrm{~T}$ we obtain,

$$
c / \bar{R}=\Sigma_{s \in \omega} y_{t s}, \quad \text { with } \quad y_{t s} \leqq x_{t s} \quad \text { for every } t, s \leqq \omega
$$


We define, by recursion, for each $t \in \omega$, a sequence $z_{t} \in{ }^{\omega}(B / I)$ as follows: We have,

$$
c \bar{R} \sum_{s \in \omega} z_{t s}^{\prime}, \quad \text { with } \quad z_{t s}^{\prime} / \bar{R}=y_{t s}, \quad \text { for every } t, s \in \omega .
$$

Then, as $\bar{R}$ is refining,

$$
c=z_{t 0}+u_{t 0}, \quad z_{t 0} \bar{R} z_{t 0}^{\prime}, \quad \text { and } \quad u_{t 0} \bar{R} \sum_{s \in \omega} z_{t, s+1}^{\prime}
$$

for every $t \in \omega$.

Repeat the same procedure to obtain $z_{t, s+1}$ given $z_{t, s}$. We have, since $\bar{R}$ is a congruence relation,

$$
\left(\sum_{s \in \omega} z_{t s}\right) \bar{R}\left(\Sigma_{s \in \omega} z_{t s}^{\prime}\right) \bar{R} c
$$

Let $d=c-\sum_{s \in \omega} z_{t s}$. Then $c / \bar{R}+d / \bar{R}=c / \bar{R}$. Thus, $h+d / \bar{R}=h$ by $1.30 \mathrm{~T}$. By 4.3 we get that $d=0 / I$.

Therefore, $c=\sum_{s \in \omega} z_{t s}$ and $z_{t s} / \bar{R}=y_{t s}$ for all $t, s \in \omega$. Now, $\wedge\left\{\vee\left\{z_{t s}: s \in \omega\right\}: t \in \omega\right\}=\wedge\{c: t \in \omega\}=c \neq 0$. Since $B / I$ is weakly $\sigma$ distributive, there is a function $\phi$ from $\omega$ into the finite subsets of $\omega$ such that,

$$
\wedge\left\{\vee\left\{z_{t s}: s \in \phi(t)\right\}: t \in \omega\right\} \neq 0
$$

So,

$$
\begin{aligned}
0 & \neq \wedge\left\{\vee\left\{z_{t s}: s \in \phi(t)\right\}: t \in \omega\right\} / \bar{R} \leqq \wedge\left\{\Sigma_{s \in \phi(t)} y_{t s}: t \in \omega\right\} \\
& \leqq \wedge\left\{\sum_{s \in \phi(t)} x_{t s}: t \in \omega\right\}=\wedge\left\{\vee\left\{x_{t s}: s \in \phi(t)\right\}: t \in \omega\right\}
\end{aligned}
$$

Therefore, the lemma is proved. We now continue with the proof of 4.2:

From (iv), 4.4 and 4.5 we obtain that $\&$ has the Kelley property and is weakly $\sigma$-distributive. Then using (Th. 9 of [10],), we obtain a strictly positive measure $\mu$ on 5 .

Let $\operatorname{Co}(X)$ be the family of open-closed sets of $X$. Define the measure $\bar{\mu}$ on $\operatorname{Co}(X)$ by $\bar{\mu}(E(a))=\mu(\infty a)$. Since $X$ is compact, $\bar{\mu}$ is countably additive as a measure on the field of sets $\operatorname{Co}(X)$. Extend $\bar{\mu}$ to the $\sigma$-field $\mathbf{B}(X)$ of subsets of $X$ generated by $\operatorname{Co}(X)(\mathbf{B}(X)$ are the Borel sets in $X)$. Using normal measure-theoretic procedures, define an integral $\Pi$ on all $\bar{\mu}$-measurable bounded functions. All functions in $\mathscr{C}(X, \overline{\mathbf{R}})$ are bounded $\bar{\mu}$-measurable.

Define $\lambda$ on $\mathfrak{A}$ by, $\lambda(a)=\Pi(F(a)) . \quad \lambda$ satisfies the following properties:

(3) $\lambda(h)=1$. 
(3) is proved by,

$$
\lambda(h)=\Pi(F(h))=\bar{\mu}(E(h))=\mu(\infty h)=1 .
$$

(4) $\lambda(a+b)=\lambda(a)+\lambda(b)$, for all $a, b \in A$.

(4) is proved by,

$$
\lambda(a+b)=\Pi(F(a)+F(b))=\Pi(F(a))+\Pi(F(b))=\lambda(a)+\lambda(b) .
$$

(5) If $x \in{ }^{\omega} A$ is a nondecreasing sequence, and $a=\vee_{i \in \omega} x_{i}$, then $\lim _{i \rightarrow \infty} \lambda\left(x_{i}\right)=\lambda(a)$.

Proof of (5). Let $x \in{ }^{\omega} A, x_{\imath} \leqq x_{i+1}$ for all $i<\omega$, and $a=$ $\vee_{i<\omega} x_{i}$. Then

$$
\lim _{i \rightarrow \infty} \lambda\left(x_{i}\right)=\lim _{i \rightarrow \infty} \Pi\left(F\left(x_{i}\right)\right)=\Pi\left(\lim _{i \rightarrow \infty} F\left(x_{i}\right)\right), \text { where } \lim _{i \rightarrow \infty} F\left(x_{i}\right)=f
$$

is the pointwise limit of the functions $F\left(x_{t}\right)$ 's (this limit exists, because $F\left(x_{i}\right) \leqq F\left(x_{i+1}\right)$ for all $\left.i<\omega\right)$. We have that $E\left(x_{i}\right)$ is the support of $F\left(x_{i}\right)$. Thus, if $y \notin \cup\left\{E\left(x_{i}\right): i \in \omega\right\}$, then $f(y)=0$.

We also have that $\vee_{i \in \omega} F\left(x_{i}\right)=F(a)$, where the l.u.b. is taken in $\langle\mathscr{C}(X, \overline{\mathbf{R}}),+, \Sigma\rangle$. The support of $F(a)$ is $E(a)$. Now, $\mu(\infty a)=$ $\lim _{\imath \rightarrow \infty} \mu\left(\infty x_{i}\right)$, because $\infty a=\vee_{\imath \in \omega} \infty x_{t}$ and $\infty x_{t} \leqq \infty x_{i+1}$ for all $i \in \omega$. Thus, $\bar{\mu}(\mathbb{E}(a))=\lim _{i \rightarrow \infty} \bar{\mu}\left(E\left(x_{i}\right)\right)$. Also,

$$
\bar{\mu}\left(\cup\left\{E\left(x_{1}\right): i \in \omega\right\}\right)=\lim _{i \rightarrow \infty} \bar{\mu}\left(E\left(x_{i}\right)\right) \text {. Then }
$$

$\bar{\mu}\left(E(a)-\cup\left\{E\left(x_{1}\right): i \in \omega\right\}\right)=0, \quad$ and, thus, $\quad \lambda(a)=\Pi(F(a))=\Pi(f)$. Therefore, $\lim _{i \rightarrow \infty} \lambda\left(x_{i}\right)=\lambda(a)$ and (5) is proved.

(6) If $a \neq 0 . a \in A$, then $\lambda(a)>0$.

Proof of (6). $\quad \lambda(a)=\Pi(F(a))$ and $F(a)$ is a continuous nonnegative function, which is positive somewhere. Then, there is an open-closed set $C$ such that $F(a) \geqq \epsilon>0$ on $C$ for some $\epsilon>0$. But, as $\mu$ is strictly positive, $\bar{\mu}(C)>0$. Thus, $\lambda(a)=\Pi(F(a))>0$.

Since $\lambda$ satisfies (3), (4), (5) and (6), we apply $16.11 \mathrm{~T}$ and obtain a strictly positive measure on $\mathfrak{B} / I$. Transfering the measure to $\mathfrak{B}$ we obtain the desired properties.

5. Invariant measures on fields of sets. In this section, we apply Theorem 4.2 to measures on $\sigma$-fields of sets. Thus, let $\mathfrak{F}$ be a $\sigma$-field of sets and $G$ a group of transformations of $\mathfrak{F}$. If $\mu$ is a $G$-invariant measure, $\mu$ has to vanish on all $\simeq_{G}$-negligible sets. We call these sets $G$-negligible i.e. $A \in F$ is $G$-negligible if there is a sequence of 
disjoint elements $Y \in{ }^{\omega} F$, such that $A \simeq{ }_{G} Y$, for every $i<\omega . \quad N_{G}$ is the set of all $G$-negligible sets. From 4.2 we obtain immediately:

TheOrem 5.1. Let $\mathfrak{s}$ be a $\sigma$-field of subsets of a set $X, G$ a group of transformations of $\mathfrak{F}$, and $I$ a subset of $F$. The following conditions are necessary and jointly sufficient for the existence of a countably additive, $G$-invariant, and $I$-positive measure on $\mathfrak{F}$ :

(i) $\quad I$ is a proper $\sigma$-ideal in $\mathfrak{F}$.

(ii) If $A \in I$ and $B \sim_{G} A$, then $B \in I$.

(iii) $N_{G} \subseteq I$.

(iv) $\mathfrak{i} / I$ has the Kelley property and is weakly $\sigma$-distributive.

It is easy to generalize 5.1 to the case when the measure $\mu$ is required to be equal to one, not on $X$, but on another set $C \in$ $F$. Instead of considering $G$-negligible sets, we have to consider $G$ negligible sets relative to $C$ (i.e. $A \in F$ is $G$-negligible relative to $C$ if there is a sequence of disjoint elements $Y \in{ }^{\omega} F$, such that $Y_{t} \subseteq C$ and $B \simeq{ }_{G} Y$, for every $\left.i<\omega\right)$. Also, $C$ should not belong to $I$. The conjecture that the only necessary and sufficient condition for the existence of a $G$-invariant measure is that $X$ is not $G$-negligible, was proposed in [2], at least for the case when $F$ is the field of all subsets of $X$. However in [3], the following counterexample was indicated:

Let $X=\omega_{1}$ and $G$ be the group of all permutations $f$ of $X$, such that $f(x) \neq x$ for at most denumerable $x$ in $X$. It is easy to see that $G$ is a group of transformations on the field of all subsets of $X$. The ideal of $G$-negligible sets contains all sets that are at most denumerable. Thus, $X$ is not $G$-negligible. However, the existence of a $G$-invariant measure on this field would imply that $\omega_{1}$ is a measurable cardinal.

When we want measures on $B A$ 's we are mainly interested in strictly positive measures. For $G$-invariant measures $\mu$ on a $\sigma$-field of sets, it is hardly ever possible to obtain strictly positive measures, since $\mu$ must vanish on the $G$-negligible sets and, by a result of ([1], p. 194), nonempty $G$-negligible sets exist in most cases of interest. In particular, these sets exist when for every $n<\omega$, there are disjoint $Y_{0}, \cdots, Y_{n-1} \in F$ such that


ures that only vanish at $G$-negligible sets. We call these measures $G$-strictly positive (i.e. $\mu$ is $G$-strictly positive iff $\mu$ is $N_{G}$ positive). Using 4.1 we get as a particular case of 5.1:

THEOREM 5.2. Let $\mathfrak{i}$ be a $\sigma$-field of subsets of a set $X$ and $G$ a group of transformations of $\widetilde{\wp}$. The following conditions are necessary and jointly sufficient for the existence of countably additive, $G$-strictly positive, and $G$-invariant measure on $\mathfrak{s}$ :

(i) $X \notin N_{G}$;

(ii) $\mathfrak{r} / N_{G}$ has the Kelley property and is weakly $\sigma$-distributive. 


\section{REFERENCES}

1. R. Bradford, Cardinal addition and the axiom of choice, Ann. Math. Logic, 3, (1971), 111-196.

2. R. Chuaqui, Cardinal algebras and measures invariant under equivalence relations, Trans. Amer. Math. Soc., 142 (1969), 61-79.

3. The existence of an invariant measure and paradoxical decompositions, Notices Amer. Math. Soc., 20 (1973), A-636, Abstract \# 73T-B313.

4. — A semantical definition of probability, in Non-Classical Logics, Model Theory and Computability, Arruda, da Costa and Chuaqui (eds), North-Holland Pub. Co. (1977), 135-167.

5. P. C. Deliyannis, Group representations and cardinal algebras, Canad. J. Math., 22 (1970), 759-772.

6. P. A. Fillmore, The dimension theory of certain cardinal algebras, Trans. Amer. Math. Soc. 117 (1965), 21-36.

7. E. Hopf, Theory of measures and invariant integrals, Trans. Amer. Math. Soc., 34 (1932), 373-393.

8. A. Horn and A. Tarski, Measures in Boolean algebras, Trans. Amer. Math. Soc., 64 (1948), 467-497.

9. O. G. Jorsboe, Set transformations and invariant measures, Various Publications Series No. 3, Matematisk Institut, Aarhus Universitet. (1966).

10. J. L. Kelley, Measures on Boolean algebras, Pacific J. Math., 9 (1959), 1165-1178.

11. D. Maharam, On measures in abstract sets, Trans. Amer. Math. Soc., 51 (1942), 413-433.

12. - An algebraic characterization of measure algebras, Amer. Math., 48 (1947), 154-167.

13. R. Sikorski, Boolean algebras, 3rd. ed. Springer-Verlag, Berlin-Heidelberg (1969).

14. A. Tarski, Cardinal algebras, Oxford U. Press, New York, (1949).

Received August 16, 1976.

Universidade Estadual de Campinas (Brasil)

AND

Universidad CATOlica de CHILE

VICIMA MACKENNA 4860

SANTIAgo 10, Chile 



\section{PACIFIC JOURNAL OF MATHEMATICS}

\section{EDITORS}

RichaRd ARENS (Managing Editor)

University of California

Los Angeles, CA 90024

\section{R. A. Beaumont}

University of Washington

Seattle, WA 98105

C. C. Moore

University of California

Berkeley, CA 94720
J. DugunduI

Department of Mathematics

University of Southern California

Los Angeles, CA 90007

R. Finn AND J. Milgram

Stanford University

Stanford, CA 94305

\section{ASSOCIATE EDITORS}
E. F. BECKENBACH
B. H. NeumanN
F. WOLF
K. YoshidA

\section{SUPPORTING INSTITUTIONS}

UNIVERSITY OF BRITISH COLUMBIA

CALIFORNIA INSTITUTE OF TECHNOLOGY

UNIVERSITY OF CALIFORNIA

MONTANA STATE UNIVERSITY

UNIVERSITY OF NEVADA

NEW MEXICO STATE UNIVERSITY

OREGON STATE UNIVERSITY

UNIVERSITY OF OREGON

OSAKA UNIVERSITY

\author{
UNIVERSITY OF SOUTHERN CALIFORNIA \\ STANFORD UNIVERSITY \\ UNIVERSITY OF HAWAII \\ UNIVERSITY OF TOKYO \\ UNIVERSITY OF UTAH \\ WASHINGTON STATE UNIVERSITY \\ UNIVERSITY OF WASHINGTON \\ AMERICAN MATHEMATICAL SOCIETY
}

The Supporting Institutions listed above contribute to the cost of publication of this Journal, but they are not owners or publishers and have no responsibility for its contents or policies.

Mathematical papers intended for publication in the Pacific Journal of Mathematics should be in typed form or offset-reproduced (not dittoed), double spaced with large margins. Underline Greek letters in red, German in green, and script in blue. The first paragraph or two must be capable of being used separately as a synopsis of the entire paper. Items of the bibliography should not be cited there unless absolutely necessary, in which case they must be identified by author and Journal, rather than by item number. Manuscripts, in duplicate, may be sent to any one of the four editors. Please classify according to the scheme of Math. Reviews, Index to Vol. 39. All other communications should be addressed to the managing editor, or Elaine Barth, University of California, Los Angeles, California, 90024.

100 reprints are provided free for each article, only if page charges have been substantially paid. Additional copies may be obtained at cost in multiples of 50 .

The Pacific Journal of Mathematics is issued monthly as of January 1966. Regular subscription rate: $\$ 72.00$ a year (6 Vols., 12 issues). Special rate: $\$ 36.00$ a year to individual members of supporting institutions.

Subscriptions, orders for back numbers, and changes of address should be sent to Pacific Journal of Mathematics, 103 Highland Boulevard, Berkeley, California, 94708.

PUBLISHED BY PACIFIC JOURNAL OF MATHEMATICS, A NON-PROFIT CORPORATION

Printed at Jerusalem Academic Press, POB 2390, Jerusalem, Israel. 


\section{Pacific Journal of Mathematics}

Vol. 68, No. 2

April, 1977

William Allen Adkins, Aldo Andreotti and John Vincent Leahy, An analogue of Oka's theorem for weakly normal complex spaces ........

Ann K. Boyle, M. G. Deshpande and Edmund H. Feller, On nonsingularly



Rolando Basim Chuaqui, Measures invariant under a group of

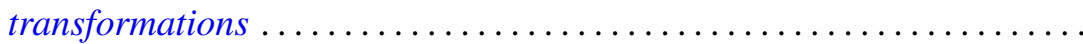

Wendell Dan Curtis and Forrest Miller, Gauge groups and classification of bundles with simple structural group .......................

Garret J. Etgen and Willie Taylor, The essential uniqueness of bounded nonoscillatory solutions of certain even order differential equations

Paul Ezust, On a representation theory for ideal systems

Richard Carl Gilbert, The deficiency index of a third order operator ........

John Norman Ginsburg, $S$-spaces in countably compact spaces using Ostaszewski's method.

Basil Gordon and S. P. Mohanty, On a theorem of Delaunay and some

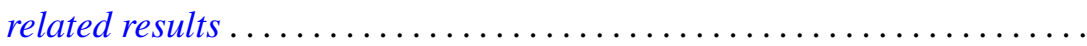

Douglas Lloyd Grant, Topological groups which satisfy an open mapping

theorem.

Charles Lemuel Hagopian, A characterization of solenoids

Kyong Taik Hahn, On completeness of the Bergman metric and its

subordinate metrics. II .

G. Hochschild and David Wheeler Wigner, Abstractly split group extensions.

Gary S. Itzkowitz, Inner invariant subspaces ...............

Jiang Luh and Mohan S. Putcha, A commutativity theorem for

non-associative algebras over a principal ideal domain.

Donald J. Newman and A. R. Reddy, Addendum to: "Rational approximation of $e^{-x}$ on the positive real axis".....

Akio Osada, On the distribution of a-points of a strongly annular function ....

Jeffrey Lynn Spielman, A characterization of the Gaussian distribution in a Hilbert space. .

Robert Moffatt Stephenson Jr., Symmetrizable-closed spaces ...

Peter George Trotter and Takayuki Tamura, Completely semisimple inverse $\Delta$-semigroups admitting principal series . . . . . . . .

Charles Irvin Vinsonhaler and William Jennings Wickless, Torsion free abelian groups quasi-projective over their endomorphism rings...

Frank Arvey Wattenberg, Topologies on the set of closed subsets ... 